for translation reinitiation or decay. Thus, the ribosome is central in scrutinizing the integrity of mRNAs in eukaryotic cells, and messages that meddle with the ribosome are shown no mercy.

1. Conti, E. \& Izaurralde, E. Curr. Opin. Cell Biol. 17, 316 325 (2005).

2. Maquat, L.E. J. Cell Sci. 118, 1773-1776 (2005).

3. Frischmeyer, P.A. et al. Science 295, 2258-2261 (2002).
4. van Hoof, A., Frischmeyer, P.A., Dietz, H.C. \& Parker, R. Science 295, 2262-2264 (2002).

5. Doma, M.K. \& Parker, R. Nature 440, 561-564 (2006).

6. Amrani, N. et al. Nature 432, 112-118 (2004).

7. Czaplinski, K. et al. Genes Dev. 12, 1665-1677 (1998).

8. Wang, W., Czaplinski, K., Rao, Y. \& Peltz, S.W. EMBO J. 20, 880-890 (2001)

9. Gatfield, D. \& Izaurralde, E. Nature 429, 575-578 (2004).

10. Onouchi, H. et al. Genes Dev. 19, 1799-1810 (2005).

11. Adham, I.M. et al. Mol. Cell. Biol. 23, 1470-1476
(2003)

12. Davis, L. \& Engebrecht, J. Genetics 149, 45-56 (1998).

13. Eberhart, C.G. \& Wasserman, S.A. Development 121 3477-3486 (1995).

14. Cougot, N., Babajko, S. \& Seraphin, B. J. Cell Biol. 165 31-40 (2004).

15. Sheth, U. \& Parker, R. Science 300, 805-808 (2003).

16. Cao, D. \& Parker, R. Cell 113, 533-545 (2003).

17. Muhlrad, D. \& Parker, R. Nature $370,578-581$ (1994).

18. Mitchell, P. \& Tollervey, D. Mol. Cell 11, 1405-1413 (2003).

\title{
Synaptotagmin: fusogenic role for calcium sensor?
}

\author{
Joshua Zimmerberg, Sergey A Akimov \& Vadim Frolov
}

\begin{abstract}
Two recent studies focusing on synaptotagmin-1's role in synaptic vesicle fusion suggest that it may be key in bringing vesicle and target membranes together and in promoting SNARE assembly. The highly positive electrostatic potential of the synaptotagmin surface could catalyze fusion.
\end{abstract}

One of the abiding mysteries in biology is the great speed of synaptic transmission, where synaptic vesicles laden with neurotransmitter fuse to the presynaptic membrane and release their content to the synaptic cleft. The $\mathrm{Ca}^{2+}$ triggered release of neurotransmitter begins some tens of microseconds after $\mathrm{Ca}^{2+}$ floods the presynaptic intracellular release site ${ }^{1}$. Thus, the mechanism of membrane fusion must account for how $\mathrm{Ca}^{2+}$ triggers the extremely fast formation of a fusion pore linking vesicular and plasma membranes that were hitherto stable and not leaky. The current paradigm for exocytotic fusion sets the trans-SNARE complex ${ }^{2}$, composed of proteins localized in vesicular and plasma membranes, as the minimal fusion machine. The calcium dependence of fusion is believed to be regulated by proteins such as synaptotagmin-1 (syt), which acts as both a 'calcium sensor' mediating $\mathrm{Ca}^{2+}$ triggering and a regulator of fusion-pore dynamics during neurotransmitter release ${ }^{3,4}$. Two recent studies $^{5,6}$, including one on page 323 of this issue ${ }^{6}$, suggest that syt may have a more central role in mediating fast synaptic fusion.

Syt is a member of a family of transmembrane proteins that sit in the vesicular membrane. Its $\mathrm{Ca}^{2+}$ sensitivity arises from its two cytoplasmic C2 domains, C2a and C2b,

The authors are at the Laboratory of Cellular and Molecular Biophysics, National Institute of Child Health and Human Development, US National Institutes of Health, 10/10D14 MSC 1855, Bethesda, Maryland 20892-1855 USA. email:joshz@helix.nih.gov
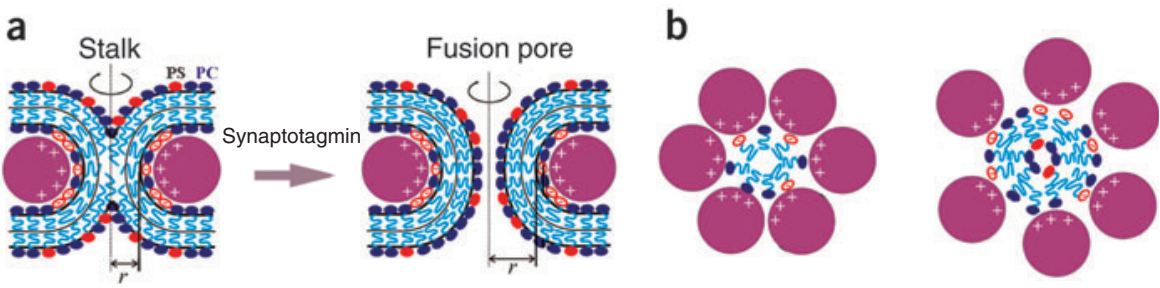

Figure 1 Synaptotagmin as a catalyst for fusion. Side (a) and top (b) views of stalk and fusion pore surrounded by six $\mathrm{C} 2 \mathrm{~b}$ domains show that synaptotagmin can energetically favor formation of both stalks and fusion pores, and may attract charged lipids in the stalk-pore membrane area. The radius, $r$, is drawn from the center of the stalk or pore laterally outward to the neutral

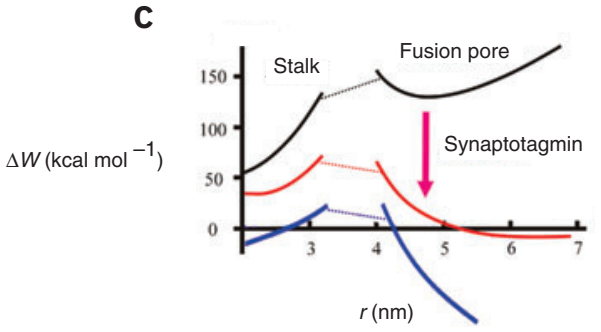
plane of the outer monolayer.

(c) Hypothetical decreases of the elastic energy of stalk and pore formation by synaptotagmin. Black, energies of the stalk and pore formed between two flat bilayers (as shown in a) separated by $\sim 3 \mathrm{~nm}$. Elastic energy of each lipid monolayer was calculated analytically using the Hamiltonian from ref. 23, assuming the stalk and pore shapes drawn in $\mathbf{a}^{22}$. Blue, stalk and pore energies when synaptotagmin electrostatically compensates the bending energy of the bound monolayer. Red, stalk and pore energies when synaptotagmin assists bending by changing the spontaneous curvature of PS (lipid composition of $30 \%$ PS and $70 \%$ PC; PC spontaneous curvature of $-1 / 8.7 \mathrm{~nm}^{-1}$ is assumed ${ }^{5}$ ). Dotted curves indicate the transition state explicitly calculated in ref. 22.

attached by a long linker to its transmembrane domain. $\mathrm{C} 2 \mathrm{a}$ and $\mathrm{C} 2 \mathrm{~b}$ have three and two $\mathrm{Ca}^{2+}$ binding sites, respectively, located in distal loops of the domains. Upon $\mathrm{Ca}^{2+}$ binding, the electrostatic potential around each $\mathrm{C} 2$ domain becomes highly positive for a large part of the domain surface, extending far beyond the $\mathrm{Ca}^{2+}$-binding loops $s^{7,8}$. As a result, both domains bind negatively charged lipids, such as phosphatidylserine (PS $)^{9,10}$. Arac et al. ${ }^{5}$. now show two important aspects of this syt-membrane interaction. Using highly purified $\mathrm{C} 2 \mathrm{a}$ and $\mathrm{C} 2 \mathrm{~b}$ domains of syt, they find that membrane adsorption induced by $\mathrm{Ca}^{2+}$ is not accompanied by any domain aggregation, previously hypothesized to facilitate fusion $^{11}$. Instead, they find that the $\mathrm{C} 2 \mathrm{~b}$ domains makes extensive interactions with liposomal membranes that involve not only $\mathrm{Ca}^{2+}$-binding loops, but also other positively charged regions (such as the polybasic region), located on the 
distal side of the domain. They further demonstrate that these regions can cause electrostatic flocculation of liposomes: positively charged C2b domains link together negatively charged liposome bilayers ${ }^{12}$. In contrast, without the $\mathrm{C} 2 \mathrm{~b}$ domain of syt, mixing complementary vesicles reconstituted with either vesicle (v-) SNAREs or target membrane ( $\mathrm{t}-$ ) SNAREs in a fusion assay $^{2}$ does not lead to either observable vesicle clustering or adhesion, even at the peak rate of lipid mixing ${ }^{13}$.

To explain the extensive coverage of the $\mathrm{C} 2 \mathrm{~b}$ domain by membrane, Arac et al. ${ }^{5}$ propose that membranes bend around the domain, and thus the $\mathrm{C} 2 \mathrm{~b}$ domain may directly participate in the formation of highly bent membrane intermediates thought to be at the core of fusion. This proposal can be considered in the context of the stalk-pore theory of fusion, which is strongly supported by recent experiments (see refs. $14,15)$. Two phospholipid bilayers in close proximity start to fuse when the lipids in their contacting leaflets bend toward each other to form highly curved intermediates-first the stalk and then the pore. Syt may modulate the curvature of these membrane intermediates through electrostatically driven bending. The electrostatic energy of the $\mathrm{Ca}^{2+}$-binding loops of $\mathrm{C} 2 \mathrm{~b}$ adsorbing onto a flat membrane is estimated at several $\mathrm{kcal} \mathrm{mol}^{-1}$ (ref. 8). Six syt molecules (Fig. 1a,b) could dramatically reduce the barrier for stalk formation if the electrostatic binding energy were used to compensate the bending energy of the bound lipid monolayer (Fig. 1c). With stalk-widening and pore formation, more charged lipids are available for syt, so the proteins effectively pull the curved membrane out to expand the stalk and pore.

The above estimates predict that syt both initiates fusion and regulates fusion-pore dynamics. In particular, pulling by syt would promote expansion of fusion pores that would otherwise stay narrow in a transient mode of vesicle fusion called kiss-and-run release (Fig. 1). Recent experimental data corroborate these predictions ${ }^{4}$. Curiously, PS changes its spontaneous curvature from $+1 / 14.4 \mathrm{~nm}^{-1}$ to $-1 / 2.3 \mathrm{~nm}^{-1}$ when fully protonated ${ }^{16}$, enough to explain the compensation of bending energy by syt described above (Fig. 1). Although protonation of free PS in physiological solution is highly unlikely, one can imagine ways that tight apposition with syt might discharge the lipid headgroups and dehydrate the headgroup region to some extent, thus effectively reducing the spontaneous curvature of PS.

In addition to lipid binding, syt also binds SNAREs and SNARE complexes. Balla et al. ${ }^{6}$ now suggest that synaptotagmin drives the assembly of SNARE complexes, a potential scaffold for $\mathrm{C} 2 \mathrm{~b}$ recruitment. They use a recon-
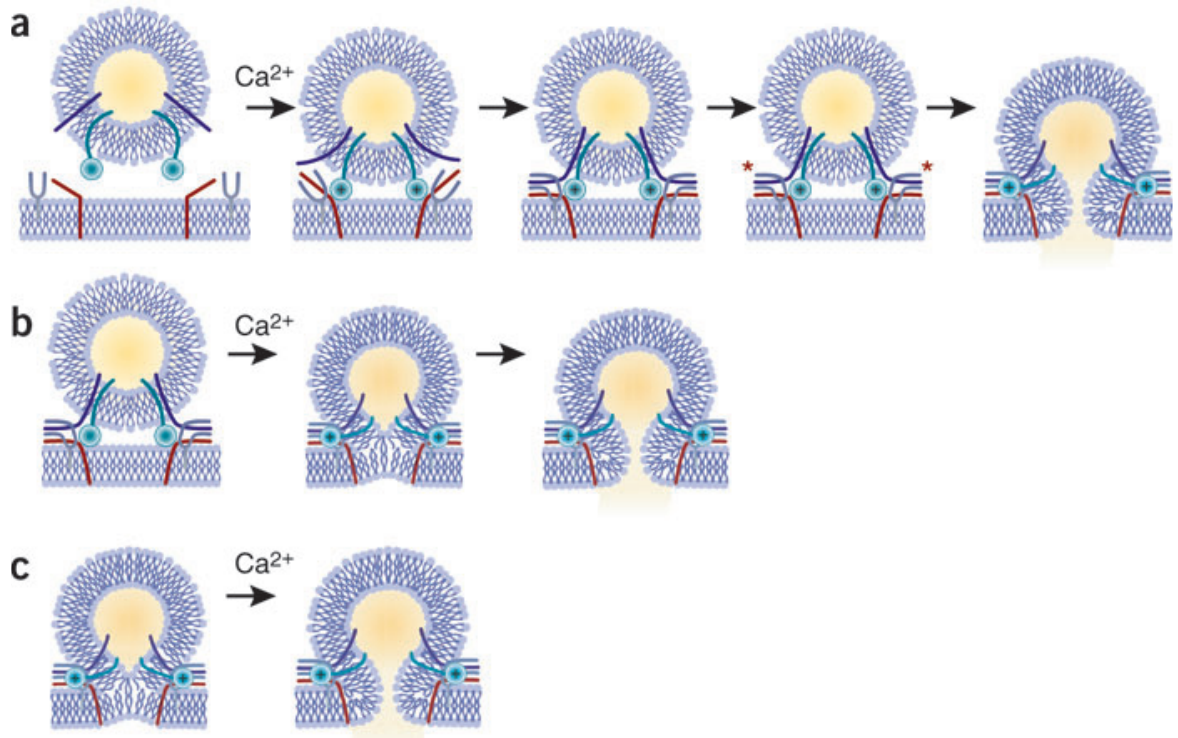

Figure 2 Possible pathways for $\mathrm{Ca}^{2+}$-stimulated membrane fusion of exocytosis. (a) 'Calcium sensor regulates the SNARE fusion machine' paradigm. Vesicles are initially tethered together by other factors (not shown). Upon chelating calcium, syt (aqua) binds PS and syntaxin (red), promoting binding of SNAP-25 (gray) and forming an attachment site for synaptobrevin (purple), which binds to form the four-helix bundle of the SNARE complex. Syt, $\mathrm{Ca}^{2+}$ and PS now drive conformational changes (asterisk) in the SNAREs to promote fusion of the vesicular membrane to the plasma membrane. $(\mathbf{b}, \mathbf{c})$ 'SNAREs are scaffolds for syt' paradigm. Docked vesicles are poised to fuse, with the SNARE complex holding and concentrating syt. Upon calcium binding, the positively charged surface of syt attracts the negatively charged membrane, binding (b) and bending the membrane to promote their initial hemifusion (the stalk) and then pulling on the membrane stalk laterally to open the fusion pore. If docked vesicles are already hemifused to the plasma membrane by SNARES, binding of $\mathrm{Ca}^{2+} \mathrm{can}^{2}$ simply pull open the pore (c). This would be the fastest way for $\mathrm{Ca}^{2+}$ to trigger fusion.

stitution system like that of ref. 2, but with negatively charged phospholipid vesicles bearing either v-SNAREs or t-SNAREs. SNAREs alone are sufficient to induce a slow lipid mixing between these liposomes that is augmented by $\mathrm{Ca}^{2+}$ and syt ${ }^{6}$. Using SNAREs from different species and different trafficking pathways, Balla et al. ${ }^{6}$ show that both the SNARE binding of neuronal syt and the acceleration of SNARE-mediated fusion by syt is species or isoform dependent, working only for neuronal exocytotic SNAREs and not for yeast SNAREs. The specificity of syt enhancement clearly suggests a syt-SNARE interaction.

Balla et al. ${ }^{6}$ further show that the functional significance of this interaction is the $\mathrm{Ca}^{2+}-$ and PS-dependent recruitment by syt of the t-SNARE component SNAP-25 to syntaxin. Next they propose that the complex of syt, $\mathrm{Ca}^{2+}$ and PS changes the conformation of the SNAREs to enhance their fusogenicity. Putting the two papers together, this suggests a pathway (Fig. 2a) in which $\mathrm{Ca}^{2+}$ entering into the presynaptic terminal first binds syt, thereby bridging phospholipids, leading to tight adhesion of vesicles to the plasma membrane, and simultaneously promoting assembly of SNARE complexes by recruiting SNAP-25. Syt, PS and $\mathrm{Ca}^{2+}$ then change the SNARE complex conformation so it can cause fusion by an unknown mechanism.

But is there time for all that? An alternative paradigm is that ring assemblies of SNAREs and syt complexes form to appropriately concentrate and orient C2b domains of syt. The ordered domains then create an electrostatic tunnel for membrane fusion ${ }^{5}$ that is extended by the polybasic linker regions of syntaxin and synaptobrevin ${ }^{17}$ (Fig. 2b,c). What is the role of calcium? First, $\mathrm{Ca}^{2+}$ turns on an 'electrostatic switch' initially proposed for the syt-syntaxin interaction ${ }^{18}$, but better suited to instantaneously stress the phospholipid bilayers of the presynaptic membrane and the synaptic vesicle for the ultra-rapid exocytosis seen in the nervous system. Second, even without syt, $\mathrm{Ca}^{2+}$ speeds up fusion of SNARE-reconstituted membranes ${ }^{19}$ considerably. Perhaps $\mathrm{Ca}^{2+}$ also has a direct role, electrostatically complexing PS headgroups ${ }^{20}$ to promote fusion between negatively charged phospholipid bilayers ${ }^{21}$.

Ultimately, syt, SNAREs and the other proteins that comprise the exocytotic fusion machine must cajole lipids to move through 
a pathway that culminates in fusion-pore opening. Our view is that exocytotic fusion follows the pathway of phospholipid-membrane fusion ${ }^{15,21,22}$. The role of proteins along this pathway is to lower the several energy barriers to membrane fusion, just as enzymes lower the energy barriers to their respective reactions. As the reaction coordinate for membrane fusion is the radius of the stalk and pore ${ }^{22}$, proteins controlling radial forces should regulate forward and backward passage through the pathway toward complete fusion. The SNARE proteins and syt are the guides that walk and pull the membrane through a bumpy stalk-pore path, with electrostatic interactions having a larger role than hitherto realized.
1. Sabatini, B.L. \& Regehr, W.G. Nature 384, 170-172 (1996).

2. Weber, T. et al. Cell 92, 759-772 (1998)

3. Fernandez-Chacon, R. et al. Nature 410, 41-49 (2001).

4. Wang, C.T., Bai, J., Chang, P.Y., Chapman, E.R. \& Jackson, M.B. J. Physiol. (Lond.) 570, 295-307 (2006).

5. Arac, D. et al. Nat. Struct. Mol. Biol. 13, 209-217 (2006).

6. Balla, A., Chicka, M.C., Tucker, W.C. \& Chapman, E.R. Nat. Struct. Mol. Biol. 13, 323-330 (2006).

7. Shao, X. et al. Neuron 18, 133-142 (1997).

8. Murray, D. \& Honig, B. Mol. Cell 9, 145-154 (2002).

9. Fernandez, I. et al. Neuron 32, 1057-1069 (2001).

10. Chapman, E.R. \& Davis, A.F. J. Biol. Chem. 273 13995-14001 (1998)

11. Wu, Y. et al. Proc. Natl. Acad. Sci. USA 100, 2082 2087 (2003).

12. Damer, C.K. \& Creutz, C.E. J. Biol. Chem. 269 ,
31115-31123 (1994).

13. Chen, X. et al. Biophys. J. 90, 2062-2074 (2006).

14. Zimmerberg, J. \& Chernomordik, L.V. Science $\mathbf{3 1 0}$ 1626-1627 (2005)

15. Chernomordik, L.V. \& Kozlov, M.M. Cell 123,375-382 (2005).

16. Fuller, N., Benatti, C.R. \& Rand, R.P. Biophys. J. 85 1667-1674 (2003)

17. Weimbs, T., Mostov, K., Low, S.H. \& Hofmann, K. Trends Cell Biol. 8, 260-262 (1998).

18. Shao, X. et al. Neuron 18, 133-142 (1997).

19. Fix, M. et al. Proc. Natl. Acad. Sci. USA 101, 7311 7316 (2004).

20. Feigenson, G.W. Biochemistry 25, 5819-5825 (1986).

21. Zimmerberg, J., Cohen, F.S. \& Finkelstein, A. Science 210, 906-908 (1980).

22. Kuzmin, P.I., Zimmerberg, J., Chizmadzhev, Y.A. \& Cohen, F.S. Proc. Natl. Acad. Sci. USA 9 8, 72357240 (2001).

23. Hamm, M. \& Kozlov, M.M. 2000. Eur. Phys. J. E. 3, 323-335 (2000) 